\title{
THEORETICAL STUDY ON ADHESIVES USED IN BALLISTIC PROTECTION STRUCTURES AND TRANSPARENT ARMOR
}

\author{
Luminiţa-Cristina ALIL \\ aegyssusatm@yahoo.com \\ MILITARY TECHNICAL ACADEMY, BUCHAREST, ROMANIA
}

\begin{abstract}
This article is a brief study conducted on adhesives currently used for manufacturing performance ballistic protection structures. The study landmarks several aspects, such as: types of connections available to achieve multilayer structures based on ceramics, metals and polymers; ways in which adhesives influence the ballistic performance of protective structures; analysis of various types of adhesives used in ballistic protection industry; general considerations in the selection of adhesives for certain types of armor and protective structures; considerations for characterizing, testing and modeling adhesives
\end{abstract}

\section{Keywords}

Adhesives, ballistic protection, transparent armor

\section{Introduction}

The necessity to counteract new and newer types of threats, correlated with the need of increasing ergonomics for the ballistic protective equipment, led to the manufacture of multilayer structures, which combine features of different types of materials to achieve a high performance. The way in which these materials are combined with each other is the key to adress the design and optimization of multilayered ballistic protection structures. One of the most commonly used methods for achieving optimal joint is using the adhesive interlayers. Such combinations may be realised for both common composite armor and transparent ballistic windows.

Next, defining the behavior, testing and modeling of the adhesive interlayers in composite armor are crucial for their further development and improvement. Studies made about ordinary armor, as well as about transparent armor, indicate that the adhesives do influencethe ballistic behavior of the composite structure [1]. While the results and information about manufacturing and material properties are generally kept secret, the articles published on this subject indicate that adhesives are commonly used 
in armor, along with information on the level of development and modeling capabilities of such materials. Most often used adhesives for ballistic protection purposes are the polymers, both thermoplastic and thermoset.

In the ballistic protection field, the main role of adhesives is to maintain the homogeneity of the armor before and after impact, and for absorbing the shock through deformation and delamination. These functions require an intermediate contact layer between the adhesive and tough components (glass, ceramic, metal) - this needs to be an interface neither too weak, nor too strong.

In transparent armor, adhesives must absorb little or no light at all, because any absorption will decrease the transparency of such a composite armor. This applies not only to visible light waves, but also to near infrared range corresponding to certain sensors and night vision devices.

\section{Joints for Different Classes of Materials}

Within the ballistic protection equipments industry, the adhesives serve to the main purpose of uniting or combining two different types of material, in order to ensure the homogeneity, intregrity, and to maintain the original properties (such as transparency). To fully understand the mechanism of adhesive bonding, it is necessary to know that using adhesives for the combination of different types of materials in multilayeredballistic structures is not the only choice, but there are countless joining methods and techniques, and choosing the right one is based on several criteria, such as the type of materials to be combined, their properties, and the properties of the multilayered structure to be obtained.

Joining different types of materials such as ceramics, metals, polymers and composite materials - is used in armor systems to meet the requirements set by the ballistic threats. Therefore, the quality of joints between the different materials play an important role in the performance of the armor structure, since it must prove resistance to dynamic loads due both to impact and explosion. Increasing the number of layers of different materials used in the same ballistic structure leads to increasing the number of adhesive layers, which further leads to stringent requirements for improved performance. Current methods of joining different materials are somewhat empirical and based on the experience with other products which means that it takes systematic research efforts to understand how does the ballistic performance depend on the joining characteristics.

Joining the materials is not always easy. Generally, the joining methods are chosen according to the natureof the materials to be mixed, their geometrical configuration and the performance requirements. Moreover, greater attention is needed in joining different materials to minimize discrepancies in terms of structure and structural discontinuities. The key to achieving optimal joints between materials of different nature, which have different characteristics and bonding properties, is designing a buffer interface in order to neutralize the inconsistencies.

When joining surfaces, special attention is needed around the porosity and surface contamination. When superposing two surfaces, the effective contact area is smaller than the apparent contact area, because of the atomic-scale defects - roughness and lack of planarity - which is valid for any type of surface. This inherent roughness can be corrected by deformation or surface diffusion (direct bonding) or by infiltrating a welding agent between the two surfaces (indirect bonding). Within the armor systems, materials such as ceramics (for example, alumina, silicon carbide or boron carbide) are connected with metals (steel, aluminum, titanium or magnesium), which are further jointed with fibers or polymeric structures, by various indirect or mechanical bonding processes.

Selecting a specific technique for manufacturing a certain kind of adhesive is based on a number of factors:

- the types of materials to be joined; 
- desired function of the joint component - for example, resistance;

- operational temperature;

- applied mechanical stress (static and dynamic) on the joint;

- the tightness of the joint;

- the component design;

- costs.

Normal mechanical joints have weak bonding strength (10 to $50 \mathrm{MPa}$ ) and create stress in the ceramic surfaces, limiting flexibility in design. Their use in armor applications is thus limited, except where such conditions can be tolerated. Stress or mass transfer may occur in both direct, as well as indirect bonding. In indirect bonding applications, there is a need for an extra intermediate layer of filler alloy to joint two different materials. Examples of indirect bonding include welding, brazing, gluing and other processes involving the creation of contact between surfaces via intermediate layers. By contrast, direct bonding uses no fillers, the contact being achieved by means of processes involving deformation and diffusion between surfaces.

Diffusion bonding requires prolonged exposure to high temperature, while the deformation requires relative movement of the surfaces in contact, simultaneously applying substantial stresses. Thus, although direct bonding methods lead to obtaining strong joints, these may not be suitable for most armor applications because different types of materials have different temperature tolerances and deformation characteristics. The role of the temperature in joining different materials from a given set of surfaces is also an important operational parameter in the selection of the adhesive environment. Most joint processes involve heating the surfaces which, during cooling, develop residual stress due to the mismatch between the elastic modulus and the coefficient of thermal expansion (CTE).

Most often, combining ceramic materials with metals in armor applications is achieved by indirect bonding processes, especially by using adhesives. Although joining by epoxy adhesive is carried out under normal ambient conditions and is suitable for most materials, it is, however a relatively weak joint, compared to welding or difussion. Moreover, the low modulus of the epoxy resin leads to a great disparity in elastic impedance between the ceramic and metal, leading to poor ballistic performance. Very few adhesives have impedance close to that of metals or ceramics, because the speed of sound in the material depends on the elastic modulus and density of the material. The adhesives with such impedance closest to that of ceramics and metals are the ceramic adhesive with high melting temperature characteristics. However, these adhesives are not as strong as polymer adhesive, therefore they are often used as a backup joint method for mechanical joints. A solution for improving the performance would be combining the ceramic adhesives with polymer or other types of adhesives.

High elastic modulus interfaces may be obtained, tipically, by welding or brazing, which reduces the incompatibilities between ceramic and metal substrates. Brazing or welding metals with ceramics requirea metalic or alloy thin layer which will react with both materials to provide a solid joint. However, subjecting the surfaces to high temperatures can result in residual stresses on cooling due to the gap between the elastic modules and the coefficients of thermal expansion. The heating temperature of brazing alloys is above $450^{\circ} \mathrm{C}$, and bellow this value for welding materials. It is important to minimize the residual stresses, while seeking to obtain a high quality joints.

\section{General Considerations in the} Selection of Adhesives

In the selection process of adhesive materials used as intermediate layers in armor and transparent armor applications several material properties are higly considered. These include the strength of bonds between the various layers of armor, not only from the chemical point of view, but also considering the surface porosity, environment stability conditions, mechanical impedance, mechanical properties at different 
deformation speeds and transparency in the visible and infrared, to name just a few aspects. Figure 1 illustrates a cross-sectional view coresponding to a common transparent composite structure composed of a ceramic ballistic plate, an area of alterning thick glass and adhesive layers plus a transition area composed of thick plastic (such as polyurethane), and an absorption region, usually a polycarbonate.

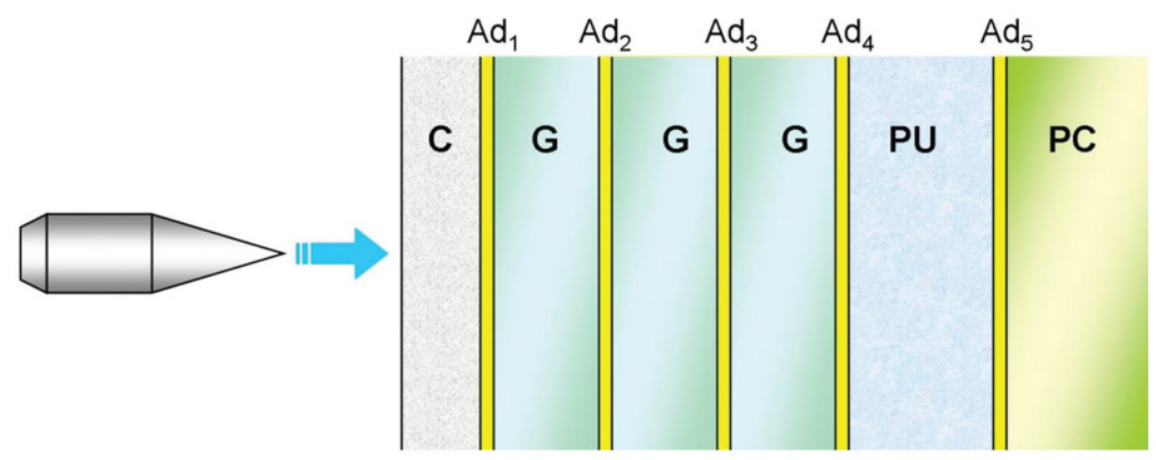

Figure no. 1 A composite structure consisting of a transparent ceramic layer $(C)$, adhesive interstrature $(A d)$, glass $(G)$, polyurethane $(P U)$ and polycarbonate $(P C)[2]$

\section{Adhesives Types}

Below we have a selection of the most important adhesives [2] used predominantly in manufacturing mustilayered ballistic protection structures:

A. Polyvinyl butyral (PVB) - it is a thermoplastic polymer developed in the late 1930 s, commonly used in the automotive industry (for windshields, windows). PVB is the raw material for a variety of adhesives used in multilayered ballistic structures, such as Saflex (Solutia, Inc.), Butacite (DuPont), Trosifol (Kuraray Europe), S-LEC (Sekisui Chemical) and KB (GlasNovation). PVB (PVC family) is part of polyvinyl-acetal group and is obtained by polycondensation of vinyl acetate with aldehyde. It is a whitecream-colored powder with a density approximately $1.2 \mathrm{~g} / \mathrm{cm}^{3}$, and the processing temperature is between $120-170^{\circ} \mathrm{C}$. Some features of interest include good optical transparency in contact with glass, controllable adhesion, resistance to elongation at bullet impact and good UV stability [3].

B. Thermoplastic polyurethane (TPU) is divided into two main categories: aliphatic and aromatic, depending on the raw materials from which it is synthesized. Examples of TPU: Dureflex (Bazer Material Science), Im 800 (and others from Inter Materials), Deerfield 4700 (and others from
Deerfield Urethane) and Huntsman 399. Generally, for transparent armor applications the aromatic TPU is preferred because its transparency is superior to that of aliphatic TPU. TPU enjoys greater popularity than PVB as it contains no plasticizer, a component that can attack other polymers such as acrylic or polycarbonate. TPU is produced in sheet shape. The composite structure is obtained by disposing multiple layers of material into a bag which is vacuumed and then autoclaved (pressed) to strengthen the layers.

C. Thermosetting polymers - there are types of thermosetting polyurethane that can also be used as intermediate adhesive layers in transparent ballistic structures. These types of adhesives are: polyurethane-urea elastomers [4], epoxy resin and mercaptans mixtures and clear epoxy resins.

D. Other materials - for joining materials such as sapphire and alumina or other high melting temperature materials, some compositions were studied, such as polyethylene-vinyl acetate (thermoplastic) and some types of glass and ceramic materials with low melting temperature [5]. Also, there are available some hybrid materials specially designed to interface adhesives and ballistic panels, for example 
FAENAC, a transparent plastic composite from Saint-Gobain Sully [6].

\section{Considerations for Testing,} Simulating and Modeling the Adhesives

The adhesives are generally tested as part of a composite structure, since the characteristics of interest are the combined properties. The simplest structure that can be considered is a composite ballistic ceramic plate bonded to a metal. An obvious problem underlying the choice of a specific type of adhesive is the impedance and the thickness of the layer. For example, a thin layer of a higher impedance adhesive is best for a ceramic as this way less energy will be reflected, limiting the damage of the tile. This phenomenon is explained by the fact that the incident energy will reach the metal layer faster and the compression wave reflected from that area will thus be reduced. As for the metal, it is better to use a thicker adhesive interlayer, to help the shock wave propagate in a larger volume of metal [7]. From this point of view, tools of numerical simulation of such phenomena can provide optimal solutions in design armor processes.

In the numerical example shown in Figure no. 2 we designed a simple multilayer structure composed of a ceramic plate, an intermediate layer of adhesive and an aluminum block. As seen in Figure no. 3, the adhesive layer is successfully fulfilling the functions for which it was intended, namely to maintain the homogeneity of the structure after the bullet impact, and having no visible influence on the overall performance of the ballistic protection structure. Of course, such examples are compelling on a training level. Scientifically, we need to approach deeper this types of problems. Therefore, the way different types of adhesives influence the performance of a ballistic protective structure is important not only for the overall performance of a ballistic protection system, but also to improve the quality of the materials that enter into the composition of such product, by understanding the complex related phenomena.

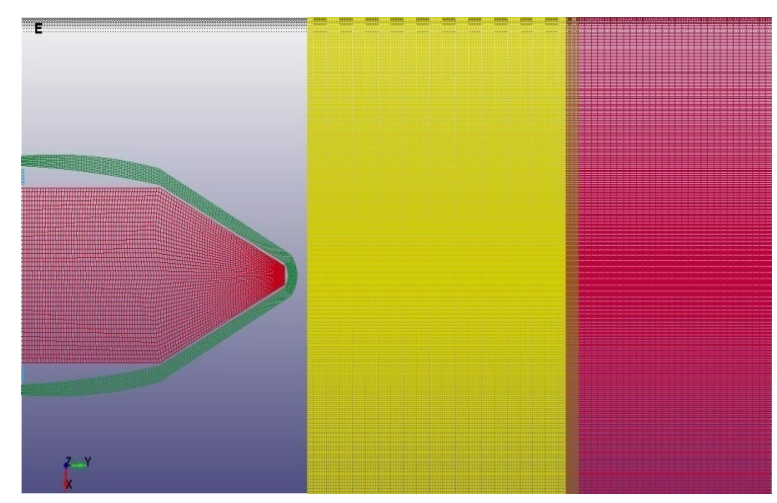

Figure no. 2 Ansys numerical model designed to illustrate the use and behavior of an adhesive layer that connects two different types of ballistic materials (in this case, ceramic and metal-aluminum)

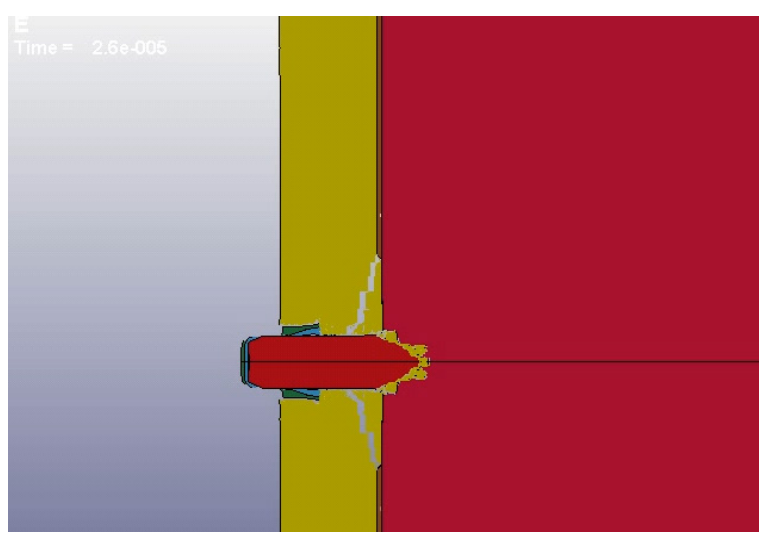

Figure no. 3 Numerical designed model that highlights the behavior of an intermediate layer of adhesive to the impact of a projectile

\section{Conclusions}

Adhesives and various other materials used as intermediate layers in manufacturing the multilayered ballistic protection structures have represented a challenge even since the invention of more complex protection structures, outlining both the boundary conditions that must be met and the structure it self. Although adhesives are successfully used in the armor systems and ballistic vests industry without special research undertaken in terms of their intrinsic qualities, it becomes increasingly clear that in order to ensure a flawless ballistic performance of the systems that contain these materials, we need to achieve a thorough understanding on how the 
adhesives do affect the ballistic behaviour of the main products. Thus, taking the risk of generating a higher production cost, we need to modernize the ballistic adhesive materials in terms of special characteristics and behaviour under high speed loads. Continuous development of simulation capabilities represents simultaneously a way to fulfill these research goals.

\section{Aknowledgement}

This paper has been financially supported within the project entitled "Horizon 2020 - Doctoral and Postdoctoral Studies: Promoting the National Interest through Excellence, Competitivenessand Responsibility in the Field of Romanian Fundamental and Applied Scientific Research", contractnumber POSDRU/159/1.5/S/140106. This project is co-financed by European Social Fund through SectoralOperational Programme for Human Resources Development 2007-2013. Investing in people!

\section{References}

1. R. Zaera, S. Sánchez-Sáeza, J.L. Pérez-Castellanos, and C. Navarro, "Modelling of the adhesive layer in mixed ceramic/metal armours subjected to impact", Composites, 31, Part A: Applied Science and Manufacturing (2000): 823-833.

2. National Research Council, Opportunities in Protection Materials Science and Technology for Future Army Applications, (Washington D.C.: The National Academies Press, 2011).

3. G.F. Freeguard, and D. Marshall, "Bullet-resistant glass: A review of product and process technology", Composites 11(1), (1980): 25-32.

4. S.S. Sarva, and A.J. Hsieh, "The effect of microstructure on the rate-dependent stressstrain behavior of poly(urethane urea) elastomers", Polymer 50(13), (2009): 3007-3015.

5. P.J. Patel, G.A. Gilde, P.G. Dehmer, and J.W. McCauley, "Transparent armor", The AMPTIAC Newsletter 4(3), (2000): 1-2.

6. http://www.saint-gobain-sully.com/pdf/quality-management/product-technical-data/ TECHNICAL-DATAS-FAENAC-FILM.pdf , accesed 15 january 2015

7. R. Zaera, S. Sánchez-Sáeza, J.L. Pérez-Castellanosa, and C. Navarro. 2000. "Modelling of the adhesive layer in mixed ceramic/metal armours subjected to impact". Composites, 31, Part A: Applied Science and Manufacturing (2000): 823-833. 